Abstract 0-004 Table 3 Aneurysm occlusion by WEB width in aneurysms with adequate WEB LC

\begin{tabular}{|c|c|c|c|c|c|}
\hline & $\begin{array}{c}\text { 4mm WEB: } \\
(\mathrm{N}=16)\end{array}$ & $\begin{array}{c}\text { 5mm WEB: } \\
(\mathrm{N}=13)\end{array}$ & $\begin{array}{c}\text { 6-7mm WEB: } \\
\qquad(\mathrm{N}=14)\end{array}$ & $\begin{array}{c}8-11 \mathrm{~mm} \\
\text { WEB:(N=8) }\end{array}$ & p-value: \\
\hline Complete & 100 & 100 & 36 & 38 & $<0.0001$ \\
\hline \multicolumn{6}{|l|}{ Occlusion, \%: } \\
\hline Adequate & 100 & 100 & 93 & 63 & 0.007 \\
\hline \multicolumn{6}{|l|}{ Occlusion, $\%$ : } \\
\hline Retreatment,\%: & 0 & 0 & 7 & 13 & 0.37 \\
\hline
\end{tabular}

nificant lower rate of complete and adequate aneurysm occlusion for larger WEB widths (table 2), even in aneurysms with adequate WEB LC (table 3). Further, there was a statisticallysignificant lower rate of retreatment in aneurysms treated with $4 \mathrm{~mm}$ and $5 \mathrm{~mm}$ WEBs (3\%) compared to 6 to $11 \mathrm{~mm}$ WEBs (18\%, p-value 0.04).

Conclusion The efficacy of aneurysm occlusion with WEB decreases with increasing WEB width despite attaining adequate WEB LC. Although attaining greater WEB LC when using larger WEBs, when technically-feasible, may increase the likelihood of complete and adequate aneurysm occlusion at follow-up, proper operator and patient expections should be set prior to treatment.

Disclosures J. Delgado Almandoz: 2; C; Microvention/Terumo. Y. Kayan: 2; C; Microvention/Terumo. A. Copelan: None. J. Scholz: None.

\section{0-005 COMPARISON OF ON-LABEL VERSUS OFF-LABEL TREATMENT OF INTRACRANIAL ANEURYSMS WITH THE PIPELINE EMBOLIZATION DEVICES}

S Cler*, D Lauzier, A Kansagra. Washington University School of Medicine, St. Louis, MO

\subsection{6/neurintsurg-2021-SNIS.5}

Background and Purpose The Pipeline Embolization Device (PED) is widely used for treatment of intracranial aneurysms, including in off-label applications. The performance of PED in off-label applications is not well understood. In this work, we quantify real-world efficacy and safety of PEDs in on-label and off-label aneurysm treatments.

Materials and Methods Clinical and angiographic data of patients who underwent PED placement at a high volume academic medical center were retrospectively obtained. Treatments were classified as on-label if they fell within the applications approved by the United States Food and Drug Administration as of 2020. Reasons for off-label classification included aneurysm location outside of approved internal carotid artery segments, size less than $4 \mathrm{~mm}$, recent rupture, and prior stent placement at the site of PED deployment. Recorded outcomes included aneurysm occlusion, procedural complications, ischemic events, in-stent stenosis, intracranial hemorrhage, post-procedural functional status, and mortality.

Results 416 aneurysms in 330 patients were treated with PED, comprising 234 on-label and 182 off-label aneurysms. Overall rate of complete aneurysm occlusion was $76.0 \%$ for on-label aneurysms and $76.3 \%$ for off-label aneurysms $(p=1.000)$. The risk of ischemic stroke in patients undergoing off-label treatments was $9.1 \%$, higher than the $4.0 \%$ in patients undergoing on-label treatment $(p=0.047)$. Procedural complications

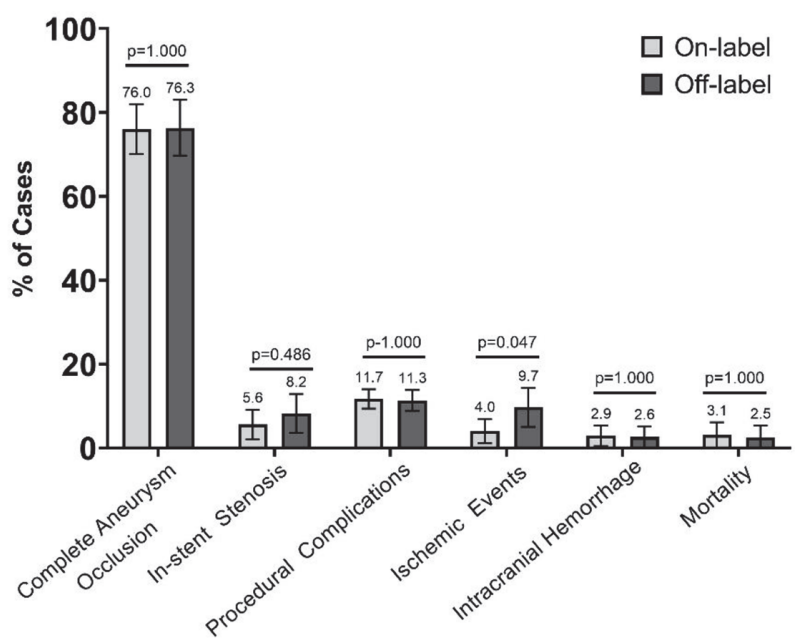

Abstract 0-005 Figure 1

including thrombus formation, vasospasm, device malfunction, access site complications, and anesthesia related complications occurred in $11.7 \%$ of on-label procedures and $11.3 \%$ of offlabel procedures $(p=1.000)$. Clinical outcomes such as intracranial hemorrhage, death, and long-term functional status were comparable between on-label and off-label groups.

Conclusion In real-world practice, off-label uses of PED can achieve similar safety and efficacy to on-label uses, though there may be a slightly higher rate of ischemic complications in off-label uses. Expert judgment is a useful supplement to official guidelines when assessing reasonable PED use beyond its approved indications.

Disclosures S. Cler: None. D. Lauzier: None. A. Kansagra: 2; C; Penumbra, Microvention, iSchemaView.

\section{0-006 PROTOTYPING A BALLOON STENT FOR MINIMALLY INVASIVE TEMPORARY ANEURYSM OCCLUSION AND EMBOLIZATION}

O Asgari*, H Berns, A Arzani, T Becker. Bioengineering, Northern Arizona University, Flagstaff, $A Z$

\subsection{6/neurintsurg-2021-SNIS.6}

Introduction Intracranial aneurysm claim 15,000 American lives annually, resulting in an additional 9,000 neurological deficits. While neuro-interventional devices rapidly advance, the use of temporary balloon occlusion to improve aneurysm sac remodeling can increase downstream ischemic risk. Balloons ensure that the aneurysm sac remains occluded during embolization, but also disrupts flow temporarily in the parent vessel.

Methods We propose an alternative to temporary balloon occlusion call the balloon-stent: an ultra-compliant urethane balloon (20 mm length, 2.5-3F diameter) encasing a selfexpandable nitinol stent that allows parent artery flow through a hollow central canal $(1.5-3 \mathrm{~mm}$ expanded diameter). The balloon-stent is deployable and retrievable, from a large primary lumen ( 0.018' ID - figure 1a). Abstract 6 Figure 1 Left $\mathrm{CAD}$ rendering of the balloon stent microcatheter device and cross-sectional view (upper right). Right - Results of a Computational Fluid Dynamic (CFD) simulation rendering velocity streamlines of an ICA aneurysm Fractional Pressure Ratio $(\mathrm{FPR})$ - pressure drop $(\Delta \mathrm{P})$ across neurovascular stenosis, is 


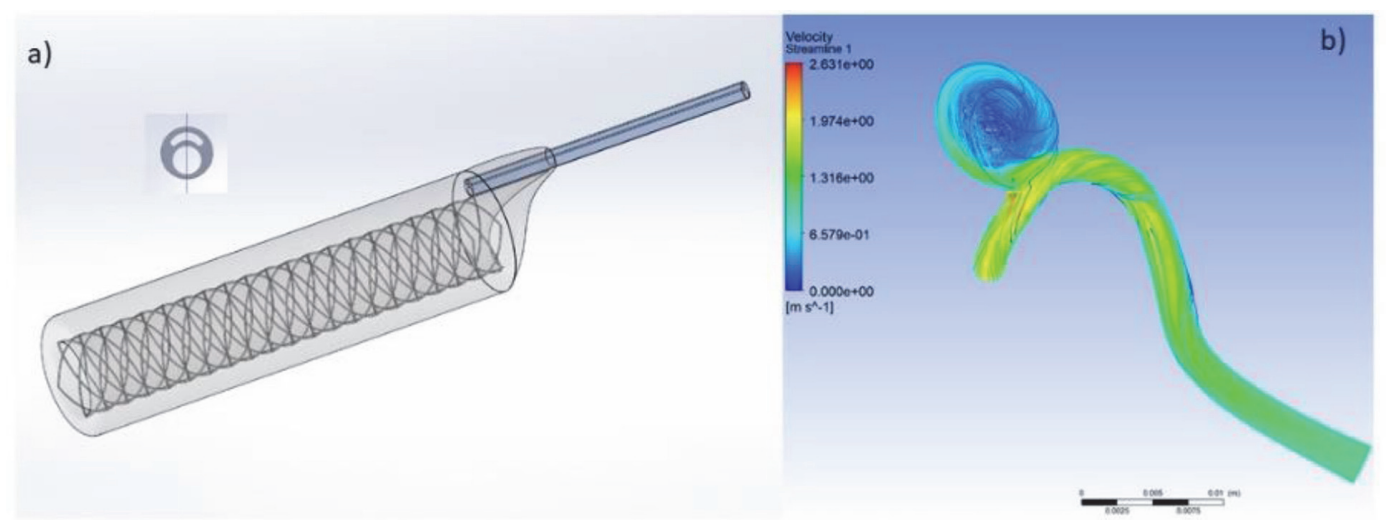

Abstract 0-006 Figure 1 a) CAD rendering of the balloon stent microcatheter device and cross-sectional view (upper right); b) results of a Computational Fluid Dynamic (CFD) simulation rendering velocity streamlines of an ICA aneurysm

derived from Fractional Flow Reserve without induced hyperemia. An FPR, with $\mathrm{a} \% \Delta \mathrm{P}<25 \%$ (equivalent to $>0.75$ FPR), is a strong indicator of patent artery flow. FPR during balloon-stent deployment was simulated using Computational Fluid Dynamics (figure 1b) and validated using benchtop modeling in a circle of Willis (CW) vessel phantom equipped with real-time branch pressure and flow monitoring.

Results A balloon-stent with a stent ID > 56\% of parent artery ID maintained $\% \Delta \mathrm{P}<25 \%$ during deployment and will minimize ischemic risk. A balloon-stent device can temporarily provide aneurysm neck protection during complementary device deployment while maintaining blood flow in the parent artery. A $2.6 \mathrm{~F}$ Penumbra Velocity ${ }^{\circledR}$, jailed next to a balloonstent device permitted an inflation ID $>56 \%$ of parent artery ID, will maintain $\% \Delta \mathrm{P}<25 \%$ during deployment and will minimize ischemic risk. The prototype maintained safe FPR and parent vessel during in vitro and CFD simulations.

Conclusion A balloon-stent device can provide neuro-interventional surgeons with a larger time-frame to deploy embolic without blood flow arrest and the need for repeated balloon inflation/deflations. In addition, this novel medical device has the potential to provide a smooth surface at the aneurysm neck for consistent device placement, minimize parent vessel trauma, eliminate ischemic effects distal to the parent artery, and minimize intra-saccular flow remnants pre- and post-treatment. Prototyping work on the balloon-stent device is currently underway.

Disclosures O. Asgari: 1; C; the 2021 Flinn Foundation Medical Technology Seed Grant. H. Berns: None. A. Arzani: None. T. Becker: None.

\section{0-007 LENGTH OF HOSPITAL STAY IN ANEURYSMAL SUBARACHNOID HEMORRHAGE PATIENTS WITHOUT VASOSPASM ON ANGIOGRAPHY: POTENTIAL FOR A FAST-TRACK DISCHARGE COHORT}

J Catapano*, V Srinivasan, K Rumalla, M Labib, C Ngueyen, T Cole, J Baranoski, C Rutledge, R Rahmani, M Lawton, A Ducruet, F Albuquerque. Neurosurgery, BNI, Phoenix, $A Z$

\subsection{6/neurintsurg-2021-SNIS.7}

Background Aneurysmal subarachnoid hemorrhage (aSAH) patients frequently suffer from vasospasm. We analyzed the association between absence of early angiographic vasospasm and early discharge.

Methods All treated aSAH patients (August 1, 2007-July $31,2019)$ at a single tertiary center were reviewed. Patients undergoing diagnostic digital subtraction angiography (DSA) on post-aSAH days 5 to 7 were included in the analysis; cohorts with and without angiographic vasospasm (angiographic reports by attending neurovascular surgeons) were compared. Primary outcome was hospital length of stay; secondary outcomes were ICU length of stay, 30-day return to the emergency department (ED) and poor neurologic outcome, defined as a modified Rankin Score (mRS) score $>2$.

Results A total of 298 patients underwent DSA on post-aSAH day 5, 6, or 7. Most patients $(n=188,63 \%)$ had angiographic vasospasm, whereas 110 patients $(37 \%)$ did not. The no-vasospasm cohort had a significantly lower mean length of hospital stay $(18.0 \pm 7.1$ days $)$ than the vasospasm group $(22.4 \pm 8.6$ days) $(\mathrm{p}<0.001)$. The 2 cohorts did not differ significantly in the percentage of patients with mRS scores $>2$ at last followup or those returning to the ED before 30 days. After adjustment for Hunt and Hess scores, Fisher grade, admission Glasgow Coma Scale score, and age, logistic regression analysis

Abstract 0-007 Table 1 Characteristics of patients with aSAH with and without angiographic vasospasm*

\begin{tabular}{|c|c|c|c|}
\hline Characteristic & $\begin{array}{c}\text { No Vasospasm } \\
(n=110)\end{array}$ & $\begin{array}{c}\text { Vasospasm } \\
(\mathrm{n}=188)\end{array}$ & P-value \\
\hline Age (yr) & $58.6(12.9)$ & $53.1(12.2)$ & $<0.001$ \\
\hline GCS on admission & $11.9(3.8)$ & $10.9(3.8)$ & 0.02 \\
\hline $\mathrm{mRS}$ at last follow-up & $2.5(2.0)$ & $3.1(2.0)$ & 0.01 \\
\hline Hunt and Hess grade & $2.8(1.1)$ & $3.1(1.1)$ & 0.01 \\
\hline Fisher grade & $3.7(0.7)$ & $3.7(0.6)$ & 0.36 \\
\hline Aneurysm size (mm) & $7.0(4.2)$ & $6.5(4.4)$ & 0.36 \\
\hline Total hospital stay (days) & $18.0(7.1)$ & $22.4(8.6)$ & $<0.001$ \\
\hline Last follow-up (days) & $835.4(1335.6)$ & $834.6(1319.0)$ & 0.99 \\
\hline Open surgical clipping, n (\%) & $55(50)$ & $122(65)$ & 0.01 \\
\hline VPS, n (\%) & $26(24)$ & $51(27)$ & 0.58 \\
\hline DSA complication, $\mathrm{n}(\%)$ & $3(3)$ & $7(4)$ & 0.64 \\
\hline
\end{tabular}

